\title{
Inferring the role of the microbiome on survival in patients treated with immune checkpoint inhibitors: causal modeling, timing, and classes of concomitant medications
}

Daniel Spakowicz ${ }^{1,2^{*+}} \mathbb{D}$, Rebecca Hoyd ${ }^{\dagger}$, Mitchell Muniak, Marium Husain ${ }^{1}$, James S. Bassett ${ }^{1}$, Lei Wang²,

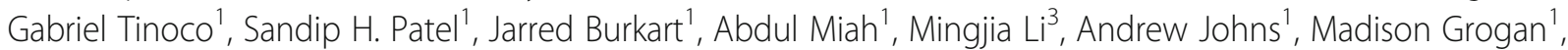
David P. Carbone ${ }^{1}$, Claire F. Verschraegen ${ }^{1}$, Kari L. Kendra ${ }^{1}$, Gregory A. Otterson ${ }^{1}$, Lang Li ${ }^{2}$, Carolyn J. Presley ${ }^{1}$ and Dwight $\mathrm{H}$. Owen ${ }^{1}$

\begin{abstract}
Background: The microbiome has been shown to affect the response to Immune Checkpoint Inhibitors (ICls) in a small number of cancers and in preclinical models. Here, we sought to broadly survey cancers to identify those in which the microbiome may play a prognostic role using retrospective analyses of patients with advanced cancer treated with $\mathrm{ICls}$.

Methods: We conducted a retrospective analysis of 690 patients who received ICI therapy for advanced cancer. We used a literature review to define a causal model for the relationship between medications, the microbiome, and ICI response to guide the abstraction of electronic health records. Medications with precedent for changes to the microbiome included antibiotics, corticosteroids, proton pump inhibitors, histamine receptor blockers, non-steroid anti-inflammatories and statins. We tested the effect of medication timing on overall survival (OS) and evaluated the robustness of medication effects in each cancer. Finally, we compared the size of the effect observed for different classes of antibiotics to taxa that have been correlated to $\mathrm{ICl}$ response using a literature review of culturebased antibiotic susceptibilities.

(Continued on next page)
\end{abstract}

\footnotetext{
* Correspondence: Daniel.Spakowicz@osumc.edu

${ }^{\dagger}$ Daniel Spakowicz and Rebecca Hoyd contributed equally to this work.

'Division of Medical Oncology, Department of Internal Medicine, The Ohio State University Comprehensive Cancer Center, Columbus, OH, USA

${ }^{2}$ Department of Biomedical Informatics, The Ohio State University College of Medicine, Columbus, OH, USA

Full list of author information is available at the end of the article
}

(c) The Author(s). 2020 Open Access This article is licensed under a Creative Commons Attribution 4.0 International License, which permits use, sharing, adaptation, distribution and reproduction in any medium or format, as long as you give appropriate credit to the original author(s) and the source, provide a link to the Creative Commons licence, and indicate if changes were made. The images or other third party material in this article are included in the article's Creative Commons licence, unless indicated otherwise in a credit line to the material. If material is not included in the article's Creative Commons licence and your intended use is not permitted by statutory regulation or exceeds the permitted use, you will need to obtain permission directly from the copyright holder. To view a copy of this licence, visit http://creativecommons.org/licenses/by/4.0/ The Creative Commons Public Domain Dedication waiver (http://creativecommons.org/publicdomain/zero/1.0/) applies to the data made available in this article, unless otherwise stated in a credit line to the data. 
(Continued from previous page)

Results: Of the medications assessed, only antibiotics and corticosteroids significantly associated with shorter OS. The hazard ratios (HRs) for antibiotics and corticosteroids were highest near the start of ICI treatment but remained significant when given prior to ICl. Antibiotics and corticosteroids remained significantly associated with OS even when controlling for multiple factors such as Eastern Cooperative Oncology Group performance status, Charlson Comorbidity Index score, and stage. When grouping antibiotics by class, $\beta$-lactams showed the strongest association with OS across all tested cancers.

Conclusions: The timing and strength of the correlations with antibiotics and corticosteroids after controlling for confounding factors are consistent with the microbiome involvement with the response to ICls across several cancers.

Keywords: Microbiome, Immune checkpoint inhibitors, Antibiotics, Corticosteroids, Cancer, Immunotherapy

\section{Background}

Treatment with Immune Checkpoint Inhibitors (ICIs) has improved patient outcomes across a wide variety of cancers. Not all patients respond to these drugs and there is a need to identify biomarkers of response. Three recent studies have shown that microbes are associated with response and overall survival (OS) in renal cell carcinoma (RCC), non-small cell lung cancer (NSCLC) and melanoma [1-3]. The microbiome may be a key player in response to ICI therapy and a potential biomarker of treatment response.

The microbiome is known to interact with the immune system, but how it affects response to ICIs is not fully understood. The effectiveness of ICI treatment relies on active $\mathrm{T}$-cell infiltration of a tumor; microbes have been associated with increased Tumor Infiltrating Lymphocytes in an IL12-depended manner [2]. However, other immune cells dampen response to ICIs such as myeloid-derived suppressor cells and FOXP3 \& CD4 + CD25+ T-regulatory cells, the levels of which have also been associated with the microbiome [4]. Moreover, the microbiome has been associated with another, systemic form of immune repression characterized by the production of prostaglandins [5-8].

Several medications commonly used during routine oncologic care and ICI treatment can influence inflammation pathways and/or the microbiome. Corticosteroids (CS) affect both of the aforementioned T-cell subtypes and the prostaglandin-related inflammatory pathways [9]. Additionally, antibiotics $(\mathrm{ABx})$ have a direct effect on the microbiome by killing or halting the growth of bacteria. Proton pump inhibitors (PPIs), histamine 2 blockers (H2Bs), non-steroid anti-inflammatory drugs (NSAIDs), and CS have also been associated with changes in the microbiome but, in contrast to antibiotics, this mechanism is indirect [10]. PPIs, by inhibiting gastric acid secretion, alter the $\mathrm{pH}$ of the gut and change the number and types of bacteria that pass through the stomach [11]. Notably, if the taxa enriched by the PPIinduced $\mathrm{pH}$ change are also important for response to
ICIs, then PPI treatment during ICI may influence clinical outcomes. The effect of other medications on clinical response may be challenging to interpret given that the effects may influence both the microbiome and ICI response.

In order to disentangle these complex interactions, we created a model of the relationship between patient characteristics, medications that affect the microbiome, inflammation, and survival. Second, we performed a retrospective analysis of patients who received ICI therapy for advanced cancer between 2011 and 2017 including medications with known effects on either the microbiome or its pathway toward affecting ICI response. Third, we estimated the association for each medication with OS. Fourth, we analyzed the effects of medications longitudinally, in order to decouple confounding variables at different time points. Fifth, we controlled for variables that broadly describe differences in baseline statuses (e.g. Eastern Cooperative Oncology Group performance status (PS)) of individuals who received concomitant medications and those who did not. Sixth, we compared the associations across several cancers, for which the medications are prescribed in subtly different ways that can be leveraged to gain further insight into the causal effects. Finally, we related these results to the microbes shown to be enriched or depleted in individuals who respond to ICIs. The combination of these strategies gives layers of support to defining the role of the microbiome in the context ICI treatment of cancer.

\section{Methods}

\section{Causal model}

We performed a literature review of the relationship between the microbiome and response to ICIs and medications that affect the microbiome (Fig. 1, references in Figure S1). From these references, a causal model was then constructed such that the nodes correspond to observable endogenous variables $\left(V_{i}\right)$, as a subset of a set of $U$ exogenous and unobserved variables that affect the 


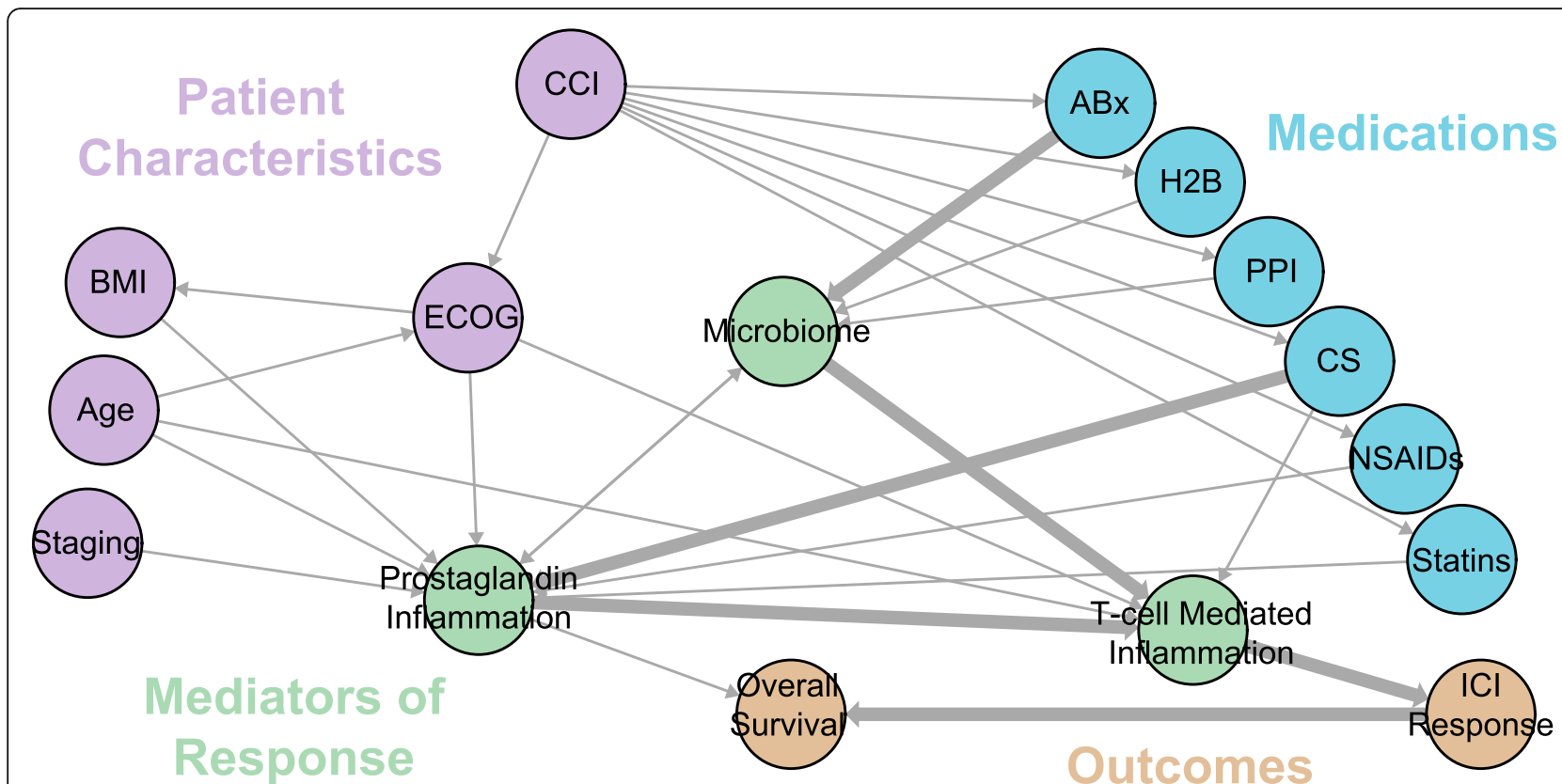

Fig. 1 Causal model for the effect of concomitant medications on Immunotherapy Response and Overall Survival. Numbers along edges refer to references supporting the connection. Hypothesized dominant pathways are shown in heavily-weighted edges [1-3, 9-32].

relationship between the microbiome and OS in patients treated with ICIs. Directed edges denote a relationship between variables when the following conditions are met: (1) there is a reported relationship between variables in which both variables were either observed or defined by intervention, and (2) the relationship cannot be explained through using an existing path. For example, Gopalakrishnan et al. reported a correlation between the microbiome and ICI response (1). This relationship exists in the graph as mediated by the nodes Microbiome $\rightarrow$ T-cell Mediated Inflammation $\rightarrow$ ICI Response, therefore no edge is drawn directly from Microbiome $\rightarrow$ ICI Response. The resulting directed acyclic graph was constructed using the igraph and dagitty packages in $\mathrm{R}[33,34]$.

\section{Retrospective data collection}

We identified patients with advanced cancer treated between 2011 and 2017 at the Ohio State University Comprehensive Cancer Center/Arthur G. James Cancer Hospital (OSUCCC-James) who received at least one dose of ICIs as part of an IRB approved retrospective study (OSU-2016C0070, OSU-2017C0063). Patient data were collected and housed in REDCap [35]. Medication timing, dose and names were collected from the electronic medical record information warehouse and validated by manual chart review. Additional diagnoses prior to ICI start were manually recorded from the Problem List, Medical History, and Encounter Diagnoses in the electronic medical record and compiled using the Charlson Comorbidity Index (CCI) [36], which includes record of myocardial infarction, congestive heart failure, peripheral vascular disease, cerebrovascular disease, dementia, chronic pulmonary disease, connective tissue disease, ulcer disease, mild liver disease, diabetes, hemiplegia, moderate or severe renal disease, moderate or severe liver disease (e.g., cirrhosis with ascites), or HIV AIDS.

\section{Medication history curation}

$\mathrm{ABx}$ and CS data were retrieved from the information warehouse within 180 days of ICI start. All medications matching a comprehensive list of steroid generic and brand names were collected with dates and routes of administration. Medications were filtered to those confirmed to be administered and the results checked against a manually-curated subset of the records.

\section{Survival analysis}

Overall survival (OS) was reported in days from the initiation of ICI to the date of death or last follow-up. All univariate and multivariate analyses were conducted using the survminer package in $\mathrm{R}[37,38]$. Univariate analyses used Kaplan-Meier survival curves with logrank tests. Multivariate analyses used Cox-Proportional Hazards models, defining the hazard function for each patient $k$ as: 


$$
h_{k}(t)=h_{0}(t) e^{\left\{\sum_{t=1}^{n} \beta_{1} A+\beta_{2} S+\beta_{3} B+\beta_{4} E+\beta_{5} G+\beta_{6} T+\beta_{7} X\right\}}
$$

Where $\mathrm{h}(\mathrm{t})$ is the hazard function at time $\mathrm{t}=1$ to $n$, $A$ is a binary indicator of antibiotic use (+/ -28 days from start of ICIs), $S$ is a binary indicator of corticosteroid use (+/-28 days from start of ICIs), $\boldsymbol{B}$ is BMI, $\boldsymbol{E}$ is the Eastern Cooperative Oncology Group performance status score [1-5], $\boldsymbol{G}$ is age, $\boldsymbol{T}$ is stage and $X$ is sex. We constructed the models using the survival package and evaluated model fits using a likelihood ratio tests in $\mathrm{R}$ [39-41].

\section{Timing analysis}

A 30-day sliding window was used to evaluate the effect of medication timing on the association with OS. Patients prescribed medications within the window were compared to a cohort of individuals who were not prescribed those medications within 180 days before or after the start of ICI treatment. Kaplan-Meier survival curves were used to estimate a hazard ratio (HR) of association with each treatment window, incremented by singledays, e.g. prescribed 180-150 days before ICI start vs no prescribed medications, and then prescribed 179-149 days before ICI start vs no prescribed medications. HRs and confidence intervals were calculated in the survival package and plotted with ggplot2 in $\mathrm{R}$ [40-42].

\section{Antibiotics and corticosteroids classes}

$\mathrm{ABx}$ and $\mathrm{CS}$ were collapsed into categories by DrugBank v5.0 accession numbers [43]. HRs were estimated for medication class and cancer combinations if the total sample set included at least 20 individuals. Cox Proportional Hazards models for the effects of $\mathrm{ABx}$ and $\mathrm{CS}$ class were used to allow for simultaneous estimation of the effects of more than one class, when applicable. Plots showing prescriptions of more than one class were created with the UpSetR package in R [44].

\section{Regularized cox regression}

Regularized Cox survival models for each cancer were implemented in the glmnet and coxnet packages in $R$ $[45,46]$. We optimized the regularization parameter by coordinate descent via 10-fold cross-validation and then tested the robustness of the parameter selection and resulting covariates with 1000 bootstrap replicates of different random samples of the dataset $[45,46]$.

\section{Reproducibility}

Scripts to reproduce all figures and analyses can be found at https://github.com/spakowiczlab/co-med-io.

\section{Results}

Causal model

The relationships between clinical variables, medications, the microbiome, ICI response and OS are strongly interconnected. Our literature review to predict their relationships (Fig. 1) led to several hypotheses testable within retrospective data. First, medications that affect ICI response via the microbiome will proceed through T-cell mediated inflammation (i.e. $\mathrm{ABx} \rightarrow$ Microbiome $\rightarrow$ T-cell mediated inflammation $\rightarrow$ ICI Response $\rightarrow$ OS). Second, the use of these medications is driven by comorbidities, which must be controlled for. Here we attempt this using the Charlson Comorbidity Index (CCI) to capture and simplify several disease states [47]. NonICI-response effects on OS proceed through Prostaglandin Inflammation. For example, the path $\mathrm{CCI} \rightarrow \mathrm{ABx} \rightarrow$ Microbiome $\rightarrow$ Prostaglandin Inflammation $\rightarrow$ OS may include sepsis, through which inflammatory processes may lead to low blood pressure or multi-system organ failure and therefore OS. Third, CS and ABx may have additive effects on ICIs through a collider effect on Tcell mediated inflammation (i.e. $\mathrm{ABx} \rightarrow$ Microbiome $\rightarrow$ T-cell mediated inflammation $\leftarrow$ CS). Finally, the clinical variables of stage, BMI, and age, and medications such as CS confound the relationship between the microbiome and ICI response, mediated by Prostaglandin-based inflammation (which itself is a collider), and therefore must be controlled for in order to infer the role of the microbiome on OS (Fig. 1).

\section{Patient characteristics}

Retrospective analysis of electronic medical records from 2011 to 2017 at the OSUCCC-James identified 690 patients treated with ICIs (Table 1$)$. Most $(76.6 \%)$ had a PS of 0 or 1 and $0-1$ co-morbidities (CCI $0-1,66.7 \%)$. The most common diagnoses were melanoma (28.5\%) and non-small cell lung cancer (NSCLC) (23.4\%). Cancers represented by fewer than 20 patients were categorized as "Other" (23.4\%). The majority of patients (90\%) had metastatic disease. ICI treatments included nivolumab in $52.8 \%$ of patients, ipilimumab in $18.0 \%$ and pembrolizumab in $15.1 \%$.

\section{Microbiome and inflammation-related concomitant medication use}

Among the medications included in the causal model, $\mathrm{ABx}, \mathrm{CS}, \mathrm{PPIs}, \mathrm{H} 2 \mathrm{Bs}$, statins and NSAIDs were identified in this cohort. $\mathrm{ABx}$ were prescribed in $36 \%$ of patients within 28 days of the start of ICIs (Table 1). The most commonly prescribed $\mathrm{ABx}$ were $\beta$-lactams (Figure $\mathrm{S} 1$ ). CS were prescribed in $40 \%$ patients within 28 days of the start of ICIs. The most commonly prescribed CS were dexamethasone and prednisone (Figure S2). PPIs were prescribed in $37 \%$ of patients. Some patients received a 
Table 1 Cohort characteristics

\begin{tabular}{|c|c|}
\hline Overall $n$ & 689 \\
\hline BMI (mean (sd)) & $27.76(6.62)$ \\
\hline Age (mean (sd)) & $62.27(13.21)$ \\
\hline Sex = Male (\%) & $402(58.3)$ \\
\hline \multicolumn{2}{|l|}{ ECOG (\%) } \\
\hline 0 & $185(31.0)$ \\
\hline 1 & $272(45.6)$ \\
\hline 2 & $113(19.0)$ \\
\hline$>2$ & $26(4.4)$ \\
\hline $\mathrm{CCl}=0-1(\%)$ & $458(66.7)$ \\
\hline \multicolumn{2}{|l|}{ Cancer (\%) } \\
\hline Bladder Cancer & $32(4.9)$ \\
\hline Head and Neck Carcinoma & $42(6.5)$ \\
\hline Melanoma & $184(28.4)$ \\
\hline Non-Small Cell Lung Cancer & $152(23.5)$ \\
\hline Renal Cell Carcinoma & $65(10.0)$ \\
\hline Sarcoma & $21(3.2)$ \\
\hline Other & $152(23.5)$ \\
\hline \multicolumn{2}{|l|}{ Staging (\%) } \\
\hline 1 & $1(0.2)$ \\
\hline 2 & $4(0.7)$ \\
\hline 3 & $44(7.2)$ \\
\hline 4 & $547(90.0)$ \\
\hline Unknown & $12(2.0)$ \\
\hline \multicolumn{2}{|c|}{ Immune Checkpoint Inhibitors (\%) } \\
\hline Atezolizumab & $22(3.2)$ \\
\hline Durvalumab & $12(1.7)$ \\
\hline Durva + Tremelimumab & $6(0.9)$ \\
\hline Ipilimumab & $126(18.3)$ \\
\hline Nivolumab & $364(52.8)$ \\
\hline Nivolumab + Ipilimumab & $37(5.4)$ \\
\hline Pembrolizumab & $104(15.1)$ \\
\hline Tremelimumab & $3(0.4)$ \\
\hline Other & $15(2.2)$ \\
\hline ATB within 28 days of $\mathrm{ICl}(\%)$ & $241(35.0)$ \\
\hline CS within 28 days of $\mathrm{ICl}(\%)$ & $273(39.6)$ \\
\hline
\end{tabular}

single medication and no others during the study period, however, more frequently patients received several medications, e.g. CS with PPI and $\mathrm{ABx}$, consistent with prophylaxis for developing an ulcer or pneumonia (Figure S3). The analysis strategy first tested for an association of a medication with OS without controlling for confounding effects of other medications and then further explored those medications with strong associations.
Across all cancer types, patients who were prescribed $\mathrm{ABx}$ within 28 days of the start of ICIs showed decreased OS (Fig. 2a). This was also true of patients prescribed CS (Fig. 2b), but not of patients prescribed other medications (Fig. 2c). ABx showed a strong negative correlation with OS in RCC, NSCLC, melanoma, and bladder cancer. CS showed a strong negative correlation with OS in NSCLC, melanoma and other cancers. While other medications were not significantly associated with OS across all cancers, several showed significant associations with specific cancers. For example, H2Bs and NSAIDs associated with decreased OS in sarcoma and NSCLC, respectively. On the other hand, PPIs and Statins positively associated with OS in sarcoma. However, we observed the strongest associations for $\mathrm{ABx}$ and $\mathrm{CS}$, and therefore followed these medications in further analyses.

\section{Timing of medication use}

Next we focused on the timing of ABx and CS prescriptions and their associations with OS in each cancer, using a 30-day sliding window (see Methods). ABx showed a greater HR than CS over nearly the entire period, and both were negatively associated with OS (Fig. 3a). ABx treatment showed the highest HR more than 100 days before the start of ICIs, a second peak near day 50, and a third, lesser peak around day 0 . CS showed a single, strong peak at day 0 . We therefore focused the timing analyses around ICI day 0 to capture the largest $\mathrm{HR}$ for both $\mathrm{ABx}$ and $\mathrm{CS}$ and to best compare the results to previous findings, and then examined the effects across cancers and drug subclasses.

\section{Antibiotics and corticosteroids classes}

The effect of $\mathrm{ABx}$ on overall survival in different cancer types was not consistently associated with $\mathrm{ABx}$ class (Fig. 3b). For example, $\beta$-lactams showed the highest HR in melanoma, but vancomycin (oral) had the highest HR in head and neck squamous cell carcinoma (HNSC). In addition, the overall effect of $\mathrm{ABx}$ was sometimes associated with a single $A B x$ subclass and sometimes distributed over many. Additionally, NSCLC strongly associated with fluoroquinolone $\mathrm{ABx}$, and this effect was stronger than the effect observed for all $\mathrm{ABx}$ combined. By contrast, the combined effect of all $\mathrm{ABx}$ in melanoma was much stronger than any individual $A B x$ class. In fact, tetracycline was positively associated with OS in melanoma patients, despite the overall effect of $A B x$ being negatively associated. On the other hand, the effects of CS classes on different cancers was more consistent (Fig. 3c), though these comparisons were often limited by the sample size. Particularly with small sample sizes, confounding effects of patients receiving multiple drugs, e.g. $\mathrm{ABx}$ and $\mathrm{CS}$, may dominate associations with OS. 


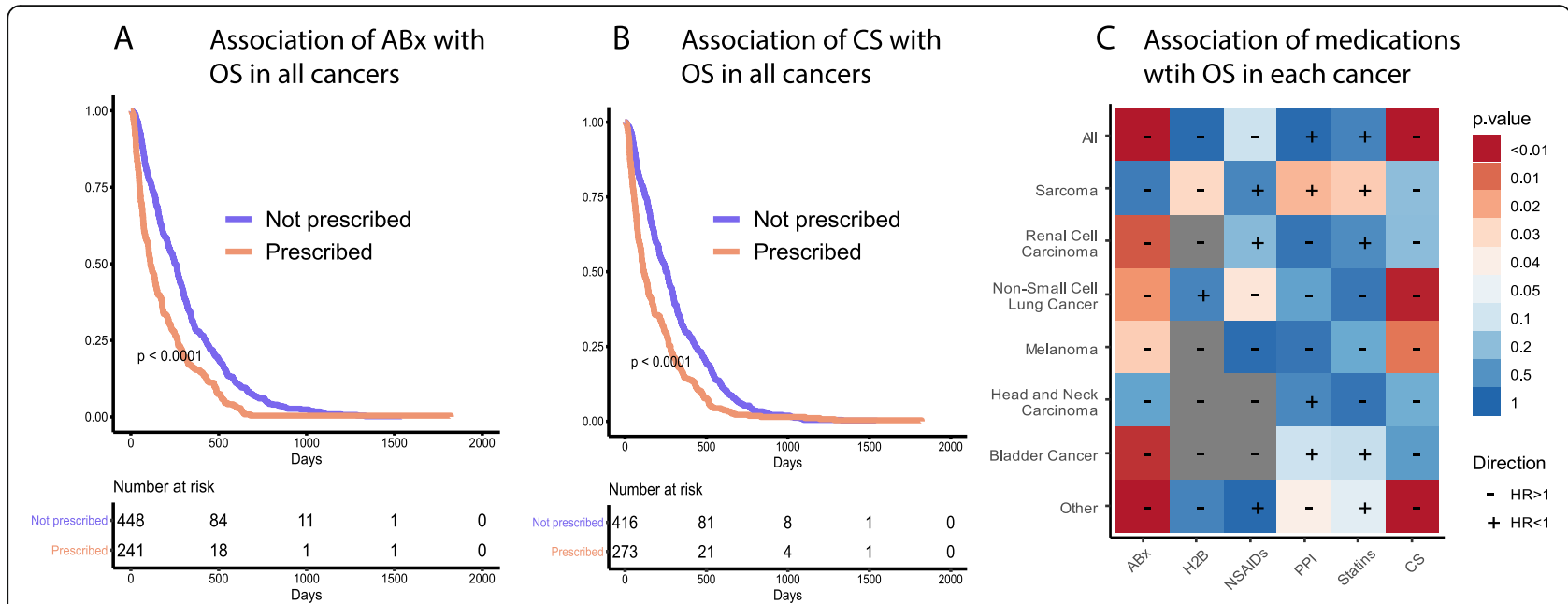

Fig. 2 The effect of medications at the start of ICI treatment across all cancers for a Antibiotics, $\mathbf{b}$ Corticosteroids, and $\mathbf{c}$ other medications. The cell color indicates the $p$-value of the Kaplan-Meier curve and the " + " or "-"the direction of the HR, in reference to its association with OS (i.e. a "-"indicates an association with decreased OS, therefore a HR > 1)

We therefore used combined models of $\mathrm{ABx}$ and $\mathrm{CS}$ to examine the effects of each.

\section{Combined modeling of $\mathrm{ABx}$ and $\mathrm{CS}$, controlling for covariates}

Models containing both $\mathrm{ABx}$ and CS showed that both are significantly associated with OS. A Kaplan-Meier curve stratifying patients by $\mathrm{ABx}, \mathrm{CS}$, or both, showed nearly identical intermediate effects of either $A B x$ or CS, and an additive combined effect (Fig. 4a). We next sought to control for confounding covariates using a
Cox Proportional Hazards model. Including CCI, PS, BMI, sex, stage, and age in the model confirmed that $A B x$ and $C S$ remained highly significant, as were PS, $\mathrm{BMI}$ and age (Fig. 4b). This suggests that $\mathrm{ABx}$ and $\mathrm{CS}$ are affecting OS in the context of ICI therapy by a mechanism other than that which is captured by PS, $\mathrm{BMI}$ or age, and is consistent with the microbiome parent to T-cell inflammation and child of $\mathrm{ABx}$ (Fig. 1).

In order to estimate the effects of $A B x$ and $C S$ within each cancer, we applied a method that (1) allowed different covariates to be included in each cancer,

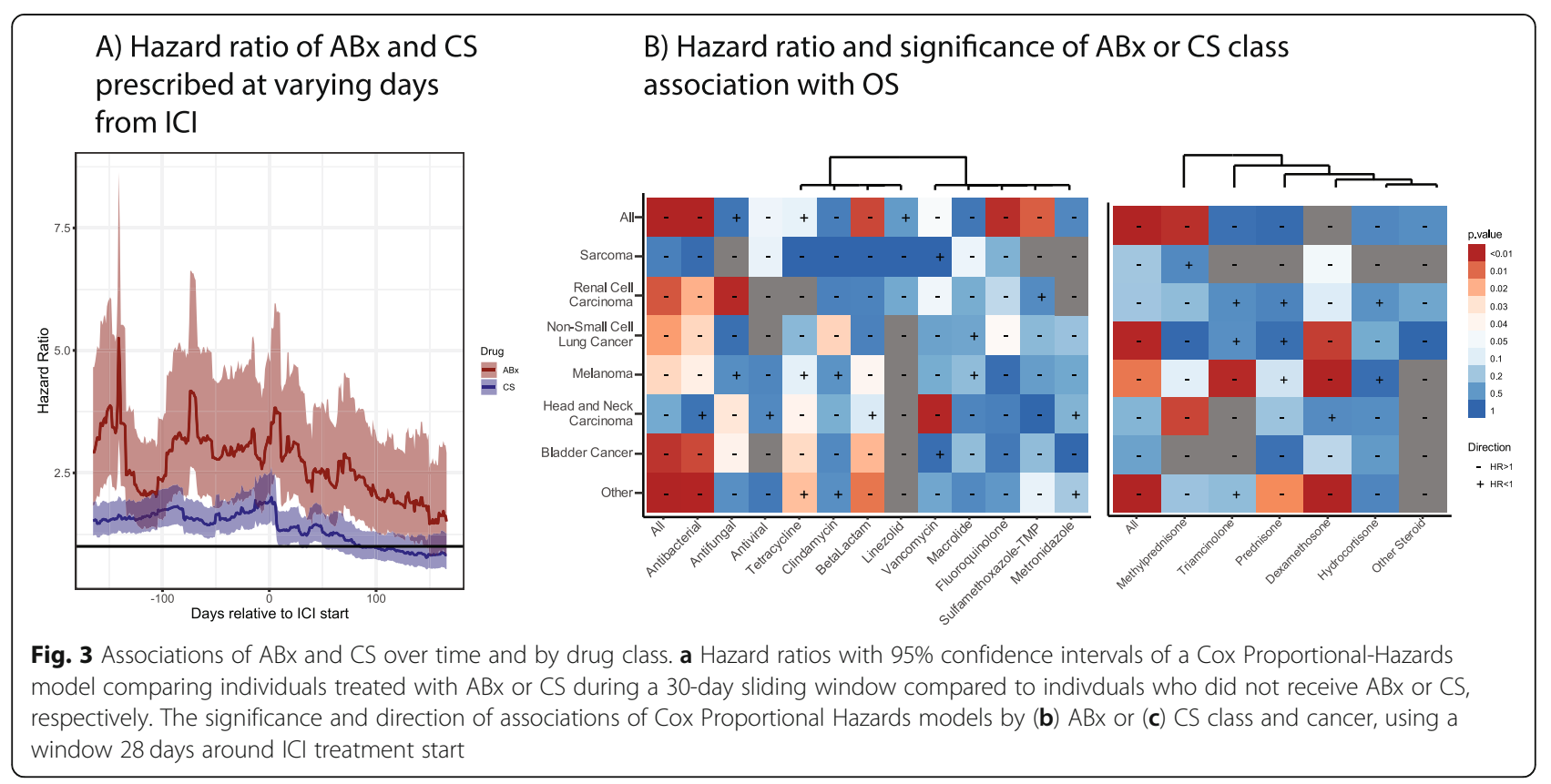


A) Survival curves comparing effects of $A B x$ and $C S$

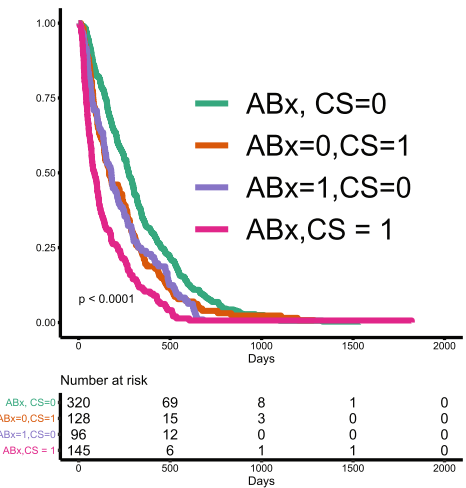

B) Combined model controlling for covariates

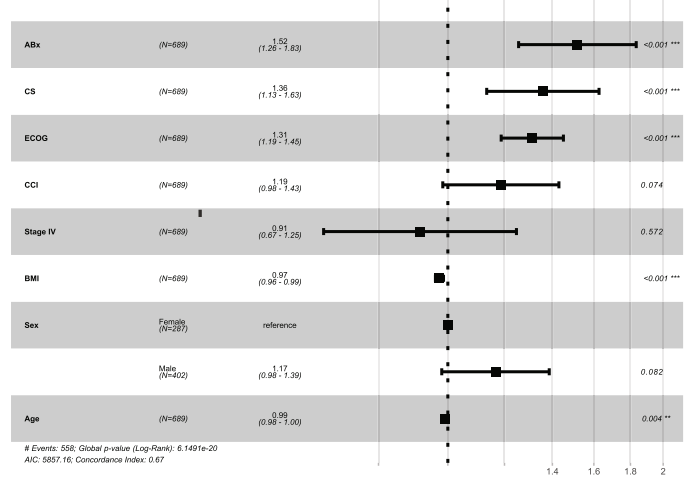

C) Cox-LASSO models with additional covariates for each cancer

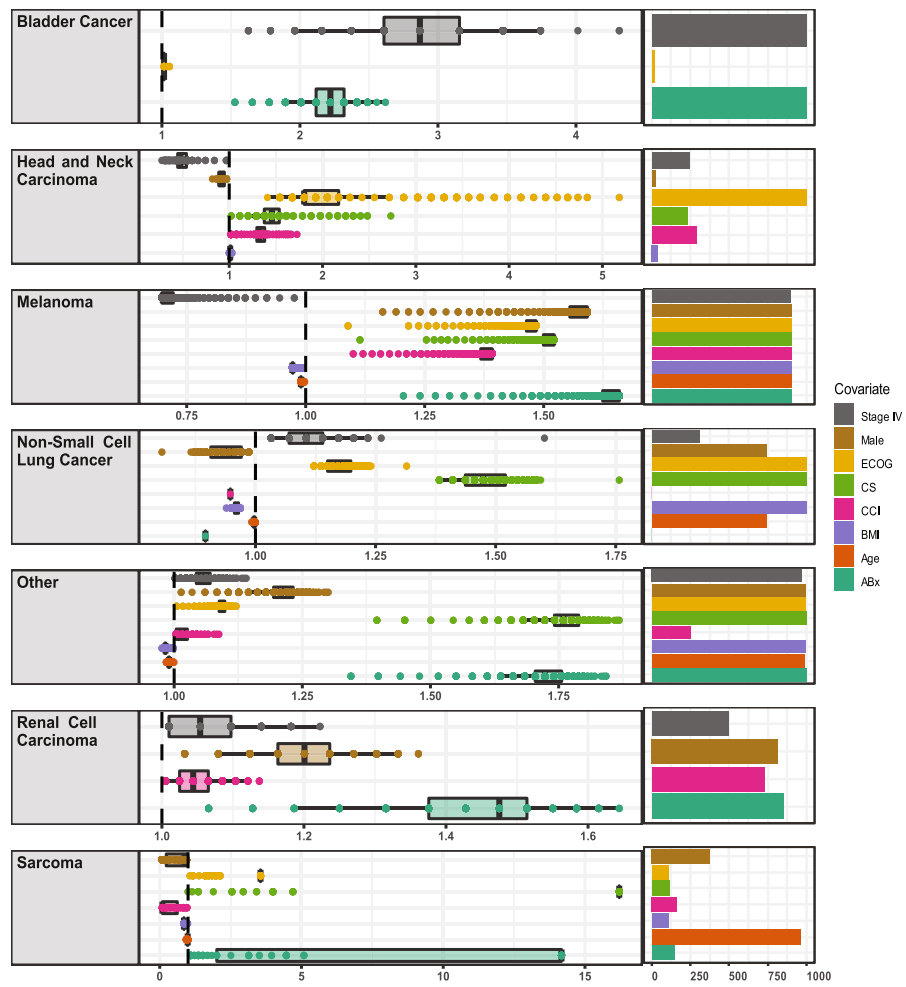

Fig. 4 Combined models for ABx and CS and controlling for covariates. a Kaplan-Meier curves for ABx and CS in combination. b Cox Proportional Hazards model incorporating both ABx and CS as well as several covariates. c Cox-LASSO models for each cancer showing the hazard ratios estimated for covariates and the number of times the covariate was included in the model. The regularization parameter was selection by 10-fold cross validation, and then the robustness was assessed by 1000 bootstrap replicates using different random samples of the data

commensurate with the different clinical features of each cancer, and (2) removed uninformative variables, increasing the power for those cancers with smaller numbers of patients in this dataset. In addition, we repeated the analysis with different random samplings of the data in order to estimate the robustness of the variable selection. We found $A B x$ to consistently and significantly associate with OS in bladder cancer, melanoma and RCC, but not in HNSC, NSCLC, or sarcoma. The HR was above 1 in each of the cancers where $\mathrm{ABx}$ was a consistently-selected covariate. Melanoma was notable in that all variables were consistently selected, with $\mathrm{ABx}$ showing the highest HRs (Fig. 4c).

\section{The relationship between $\mathrm{ABx}, \mathrm{OS}$, and the microbiome}

While no direct microbiome measurements were made in this study, we next sought to relate effects of $\mathrm{ABx}$ to the current knowledge about the organisms have been associated with ICIs. The bacterial taxa that showed the strongest enrichment in responders or non-responders to ICIs were selected from the literature and combined into a phylogenetic tree (Fig. 5) [1-3]. The taxa spanned several phyla and few ranks were consistently enriched in either responders or non-responders. For example, Firmicutes was found to be enriched in responders [1], but within the phylum are several taxa found to be enriched in non-responders [2, 3]. An exception to this was Bacteroidetes, which was found to be enriched in non-responders and each of the four species in the phylum were also enriched in non-responders $[1,2]$. We performed a literature review of $\mathrm{ABx}$ susceptibilities for each of these taxa to estimate whether the size of the $\mathrm{HR}$ of the $\mathrm{ABx}$ would relate to the taxa for which it is active. For example, an $\mathrm{ABx}$ that target only bacteria enriched in non-responders may be beneficial because it may shift the community toward those taxa enriched in responders. On the other hand, if the overall diversity of the microbiome is important, broad-spectrum ABx may have higher HRs than narrow-spectrum.

The ABx class with the largest HR across all cancers was the $\beta$-lactams. Within this group category are the cephalosporins, which have a relatively narrow spectrum of activity and a unique pattern relative to other $\mathrm{ABx}$ classes. The cephalosporins are ineffective against the 


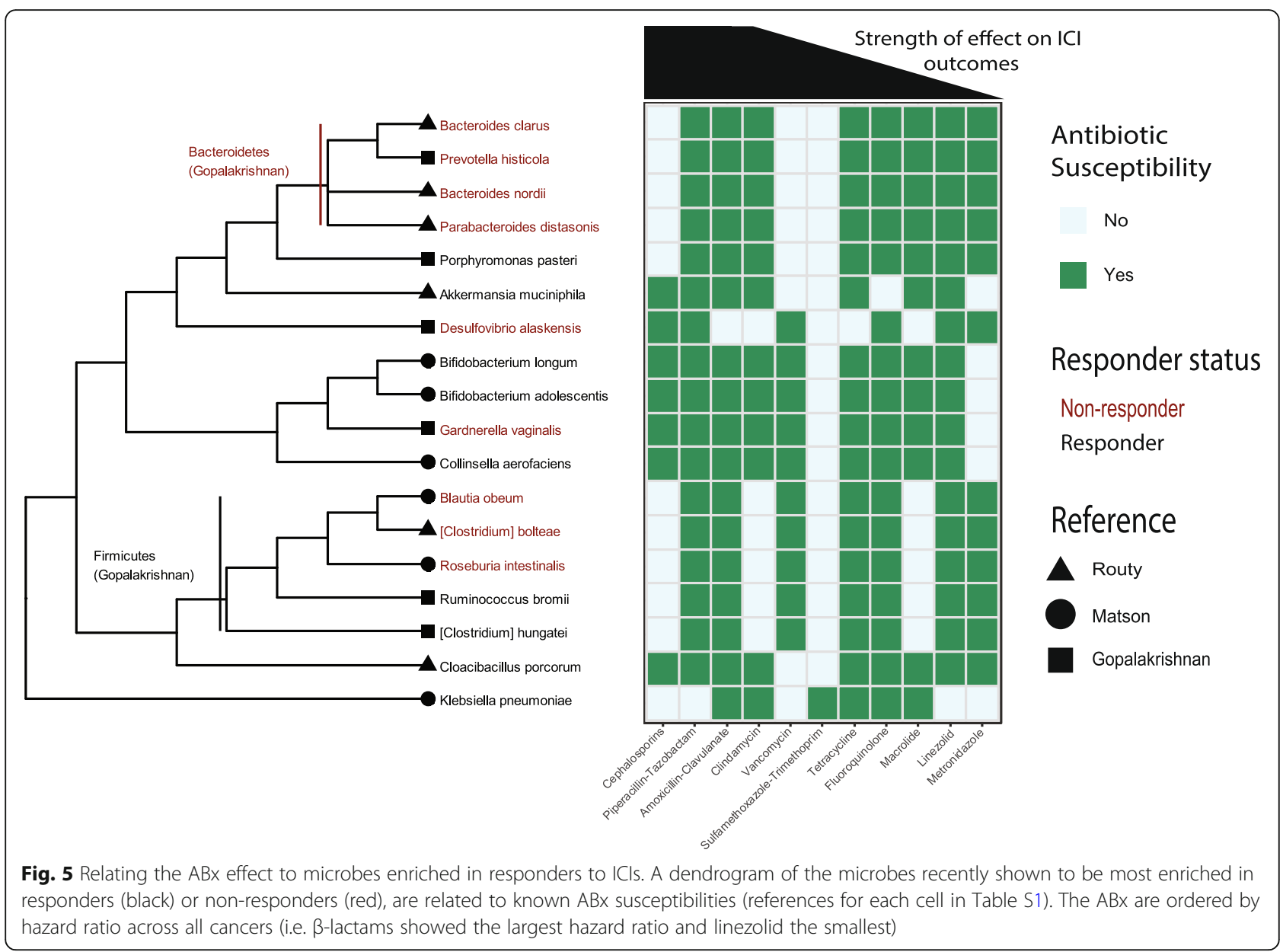

Bacteroidetes, found to be enriched in non-responders, but so were $\mathrm{ABx}$ such as vancomycin and sulfamethoxazole-trimethoprim (SXT). However, unlike vancomycin and SXT, cephalosporins effectively target A. muciniphila, which was shown to causally modify response to ICIs. Cephalosporins are also ineffective against several Firmicutes, similar to clindamycin, macrolides and metronidazole (Fig. 5).

\section{Discussion}

The effects of medications or other variables are difficult to parse in a dynamic setting such as during treatment for cancer. We used a variety of methods to show that $\mathrm{ABx}$ and $\mathrm{CS}$ are significantly associated with decreased OS in several cancer types.

The association of CS with ICI response and OS remains controversial. Our observed association is consistent with other observations of decreased OS in NSCLC [9]. However, Ricciuti et al. showed no effect of CS on OS in NSCLC when given on the same day as ICI start, when the CS was prescribed for reasons other than "cancer-related palliative indications" [48]. Our records lack some variables needed to replicate those results, however our results are consistent with aspects those findings. For example, dexamethasone treatment showed a strong negative association with OS across several cancer types, consistent with its use for brain metastases and anorexia, which are all indicators of poor clinical outcome. On the other hand, several of our analyses demonstrated associations between CS and OS that may not be due to selecting a sub-cohort with a poor prognosis. Our first causal strategy, the time analysis, showed similar results when restricting CS medications to a single day, but a larger effect when a wider time window was used (Table 2). Similar effects have been observed previously, but with little consistency in the time window tested [2, $3,9,48-52]$. Our second causal strategy, controlling for covariates, cannot be directly compared because our dataset did not include central nervous system metastases. However, when we control for metastatic stage and PS, the CS association remains. Our third causal strategy, comparisons between cancers, shows that the CS association with OS is observed in cancers for which brain metastases are not common, such as RCC, and for specific CS not typically used for brain metastases, such as methylprednisolone in HNSC. This suggests that 
Table 2 Timing of associations between medications and $\mathrm{ICl}$ response

\begin{tabular}{|c|c|c|c|c|c|c|c|c|c|c|c|}
\hline & $\begin{array}{l}\text { Cancer } \\
\text { Type }\end{array}$ & Drug Type & $\begin{array}{l}\text { Timing } \\
\text { Window (days) }\end{array}$ & $\begin{array}{l}\text { Sig } \\
\text { PFS }\end{array}$ & $\begin{array}{l}\text { Sig } \\
\text { OS }\end{array}$ & $\begin{array}{l}\text { N Drug } \\
\text { Users }\end{array}$ & $\begin{array}{l}N \\
\text { Total }\end{array}$ & $\begin{array}{l}\text { PFS } \\
\text { HR }\end{array}$ & $\begin{array}{l}\text { OS } \\
\text { HR }\end{array}$ & $\begin{array}{l}\text { Uni vs Multi } \\
\text { Variate }\end{array}$ & Controlled Covariates \\
\hline $\begin{array}{l}\text { This } \\
\text { study }\end{array}$ & Melanoma & $A B x$ & $+/-28$ & & Yes & 48 & 185 & & 1.66 & Multi & $\mathrm{CS}, \mathrm{ECOG}, \mathrm{BMI}, \mathrm{G}, \mathrm{A}, \mathrm{CG}$ \\
\hline [14] & Melanoma & $A B x$ & $(-30)-0$ & Yes & No & 10 & 74 & 0.32 & 0.52 & $\begin{array}{l}\text { Multi } \\
\text { (only for PFS) }\end{array}$ & $\begin{array}{l}\text { A, E, } G, L T, I R \text {, Serum levels of } \\
\text { lactate dehydrogenase (LDH), } \\
\text { BRAF status }\end{array}$ \\
\hline $\begin{array}{l}\text { This } \\
\text { study }\end{array}$ & NSCLC & $A B x$ & $+/-28$ & & No & 64 & 152 & & 0.81 & Multi & $\mathrm{CS}, \mathrm{ECOG}, \mathrm{BMI}, \mathrm{G}, \mathrm{A}, \mathrm{CG}$ \\
\hline [13] & NSCLC & $A B x$ & $+/-28$ & Yes & Yes & 20 & 109 & 0.29 & 0.35 & Multi & A, G, S, E, His, Mut, LT, IR, CT \\
\hline [22] & NSCLC & $A B x$ & $(-30)-0$ & Yes & Yes & 48 & 239 & 1.3 & 2.5 & Multi & A, His, S, PR, E, C, Hos \\
\hline [13] & NSCLC & $A B x$ & $(-60)-0$ & Yes & Yes & 20 & 109 & 0.29 & 0.35 & Multi & $\begin{array}{l}\text { A, G, His, S, E, LT, C, IR Mutation, } \\
\text { ABx, PPIs }\end{array}$ \\
\hline [22] & NSCLC & $A B x$ & $(-60)-0$ & No & Yes & 68 & 239 & 1.2 & 2 & Multi & A, His, S, PR, E, C, Hos \\
\hline [2] & NSCLC & $A B x$ & $(-84)-0$ & No & Yes & 37 & 140 & & 2.31 & Multi & A, G, His, S, PR, E, MS \\
\hline [2] & $\mathrm{RCC}$ & $A B x$ & $(-30)-0$ & Yes & Yes & 16 & 121 & 2.2 & 2.1 & Multi & $A, T B, R$ \\
\hline [22] & $\mathrm{RCC}$ & $A B x$ & $(-60)-0$ & Yes & No & 22 & 121 & 2.3 & 1.9 & Multi & $A, T B, R$ \\
\hline [2] & RCC & $A B x$ & $(-84)-0$ & Yes & No & 20 & 67 & 2.16 & & Multi & $A, G, R, T B$ \\
\hline [2] & UC & $A B x$ & $(-84)-0$ & No & No & 12 & 42 & 1.97 & & Multi & Hemoglobin levels, KPS, Liver M \\
\hline [49] & Several & Abx & $(-30)-0$ & & Yes & 29 & 167 & & 7.4 & Uni & \\
\hline [49] & Several & Abx & $0+$ & & No & 68 & 128 & & 0.9 & Uni & \\
\hline [49] & Several & Abx & $(-30)--$ & & Yes & 29 & 167 & & 8.2 & Multi & Cancer, E, CG, TB, A, CS \\
\hline $\begin{array}{l}\text { This } \\
\text { study }\end{array}$ & Melanoma & CS & $+/-28$ & & Yes & 66 & 185 & & 1.57 & Multi & $\mathrm{ABx}, \mathrm{ECOG}, \mathrm{BMI}, \mathrm{G}, \mathrm{A}, \mathrm{CG}$ \\
\hline [48] & NSCLC & $\begin{array}{l}\text { CS (Cancer- } \\
\text { related) }\end{array}$ & $+/-1$ & No & Yes & 66 & 650 & 1.4 & 1.6 & Multi & $\begin{array}{l}\text { A, G, S, His, LT, IR, E, Mut, Brain M, } \\
\text { PD-L1 TPS, \%, Median TMB }\end{array}$ \\
\hline [48] & NSCLC & $\begin{array}{l}\text { CS (Cancer- } \\
\text { unrelated) }\end{array}$ & $+/-1$ & No & No & 27 & 650 & 0.62 & 0.91 & Multi & $\begin{array}{l}\text { A, G, S, His, LT, IR, E, Mut, Brain M, } \\
\text { PD-L1 TPS, \%, Median TB }\end{array}$ \\
\hline [21] & NSCLC & CS & $0+28$ & Yes & Yes & 35 & 151 & 1.88 & 2.38 & Multi & $\begin{array}{l}\text { A, G, S, His, MS, E, LT, IR, Brain M } \\
\text { Bone M, Liver M, PD-L1 expression, } \\
\text { CS }\end{array}$ \\
\hline $\begin{array}{l}\text { This } \\
\text { study }\end{array}$ & NSCLC & CS & $+/-28$ & & Yes & 67 & 152 & & 1.85 & Multi & $\mathrm{ABx}, \mathrm{E}, \mathrm{BMI}, \mathrm{G}, \mathrm{A}, \mathrm{CG}$ \\
\hline [9] & NSCLC & CS & $(-30)-0$ & Yes & Yes & 90 & 640 & 1.3 & 1.7 & Multi & S, E, Brain M \\
\hline [13] & NSCLC & PPIs & $+/-28$ & No & No & 40 & 109 & 1.1 & 1.47 & Uni & \\
\hline [2] & NSCLC & PPIs & $(-84)-0$ & No & No & 35 & 140 & & & Uni & \\
\hline [2] & $\mathrm{RCC}$ & PPIs & $(-84)-0$ & No & No & 20 & 67 & & & Uni & \\
\hline [2] & UC & PPIs & -84 & No & No & 7 & 42 & & & & \\
\hline
\end{tabular}

Abbreviations: A Age, G Gender, $R$ IMDC Risk, TB Tumor Burden, His Histology, S Smoking History, PR Number of Prior Regimens, E ECOG Performance Status, C Clinical Trial, Hos Hospitalization, MS Number of Metastatic Sites, $L T$ Line of Therapy, IR ICI Regimen, CS Corticosteroids, ABx Antibiotics, CG Cancer Stage, $M$ Metastases, Mut Mutation

understanding the association between CS and the response to ICIs may require more granular assessment of CS types (i.e. rather than collapsing to $10 \mathrm{mg}$ prednisone equivalent) and cancers.

We applied the same logical framework to ABx treatment to demonstrate an effect on OS. Unlike CS, the majority of studies have found an association between $\mathrm{ABx}$ use and ICI response, independent of the time window (Table 2). Our longitudinal analysis showed a global maximum HR well before the start of ICIs, consistent with the $A B x$ effects persisting for long periods. Given this result, it is unlikely that acute illnesses drive the association between $\mathrm{ABx}$ and OS. However, a recent prospective study found that $\mathrm{ABx}$ given currently with ICI treatment did not significantly affect OS for a group of patients with lung, skin, or several other cancers [49] (Table 2). We observe lower HRs for the effect of ABx after ICI start, however it remains significant until approximately 120 days post ICI start. We note that within cancers the effect of $A B x$ is highly variable (Fig. 4c); the 
difference may be due to the composition of the cohorts (e.g. more patients with bladder cancer, where ABx has a strong effect, and fewer with NSCLC, where the effect is less). Our results are consistent with a recent metaanalysis across several cancers, in which the greatest HR was observed in the 42 days before the start of ICIs [50].

When controlling for illness-related covariates that report on the overall health status of the individual (e.g. CCI, PS) the effect of $\mathrm{ABx}$ remained significant. Third, the associations of $\mathrm{ABx}$ and $\mathrm{OS}$ were observed across cancer types (e.g. patients with bladder cancer versus melanoma). A larger fraction of bladder cancer patients were treated with $\mathrm{ABx}$ than any other cancer (56\%), consistent with their use for urinary tract or as prophylaxis for invasive urologic procedures. On the other hand, melanoma patients treated with $\mathrm{ABx}$ were the smallest fraction of any cancer (25\%), consistent with this population being less likely to undergo procedures in which prophylactic $\mathrm{ABx}$ are used. It is reasonable to suspect that melanoma patients treated with $\mathrm{ABx}$ are therefore more compromised than those not treated with $\mathrm{ABx}$. However, an effect of $\mathrm{ABx}$ remains, even for bladder cancer. Although it remains probable that the cohorts who receive $\mathrm{ABx}$ are different from those who did not in ways that have not been controlled for in analyses, these three analyses add confidence to the association of $\mathrm{ABx}$ with OS in the context of ICIs.

We next related the strength of the association of $\mathrm{ABx}$ classes with OS and the microbes that those $\mathrm{ABx}$ classes affect. The $\beta$-lactam $A B x$ were shown to have the strongest association with OS across cancer types. The literature review of antibiotic susceptibilities showed that this diverse class is effective against the Gram-positive phylum Firmicutes. The literature review of the bacterial taxa associated with response to ICIs, showed that the Firmicutes are enriched in responders to ICIs. Moreover, $\beta$-lactams are not consistently effective against members of the phylum Bacteroidetes, which was found to be enriched in non-responders. This suggests that the $\beta$ lactams may show the strongest signal across all cancers in our dataset because they disrupt the microbiome in such a way that they reduce response to ICIs by depleting the Firmicutes more so than the Bacteroidetes.

The association between $\mathrm{ABx}$ prescriptions and $\mathrm{OS}$ that we observe is consistent with direct measurements of the microbiome and response to ICIs [1-3]. However, there is no consensus for which taxa are enriched in the responders to ICIs (Fig. 5). For example, there is causal evidence for Akkermansia muciniphila increasing response to ICIs, however, it was not among the most enriched in the other datasets [1-3]. Nonetheless, some agreement can be observed between the effects of $\mathrm{ABx}$ on isolated taxa and OS. Narrow spectrum $\beta$-lactams (e.g. cephalosporins), which show the strongest association with OS, are not effective against Bacteroidetes (enriched in non-responders (1)) but are against $A$. muciniphila (enriched in responders (2)). However, we note that the effects of $\mathrm{ABx}$ can be difficult to predict over long time scales; some broad spectrum $\beta$-lactams have resulted in increased Firmicutes post-recovery, despite being effective against them [51].

The results presented here contrast with several assumptions gathered from the literature and described by the causal model (Fig. 1). First, we found that ABx and $\mathrm{CS}$ are the only medications significantly associated with OS, despite the inclusion of several medications associated with changes to the microbiome (Fig. 2). This may be due to the types of changes incurred (e.g. PPIs may not significantly change the abundances of those taxa linked to ICI response) or the strength of the effect amid the noise in the data. However, the other two hypotheses were borne out by the analyses.

The CS and ABx medications showed an additive effect on OS, consistent with a collider interaction in the model (Fig. 4a). Also, there was an effect of ABx after controlling for many covariates, consistent with its direct effect on the microbiome and the microbiome playing a role in ICIs (Fig. 4b). This result was consistent with the relationship between the strength of the $A B x$ signal and the bacterial taxa susceptible to that $\mathrm{ABx}$ (Fig. 5).

\section{Limitations}

A key challenge in this and other retrospective analyses is inferring causal relationships in non-randomized cohorts. For example, patients who receive medications such as antibiotics may be quite different from those who do not. However, it is difficult to imagine an ethical trial that could randomize treatment with $\mathrm{ABx}$ in this setting. Therefore, retrospective analyses may be the best option until direct measurements of the microbiome are widely available. We used a variety of methods to show that $\mathrm{ABx}$ and $\mathrm{CS}$ are significantly associated with decreased OS across a variety of cancers and that these results are consistent with a role for the gut microbiome.

Our study remains limited by being unable to account for important factors known to affect OS in the context of ICI treatment. For example, the complete ABx history of patients -- much longer than the windows reported here -- are very likely of consequence. Several groups have studied the recovery of microbiome diversity following $\mathrm{ABx}$ exposure and results show reasonable recovery 90 days later $[51,52]$. However, multiple courses of $\mathrm{ABx}$ prevented such a recovery; i.e. diversity returned to baseline after one treatment with $\mathrm{ABx}$, but not after a second $A B x$ treatment within 60 days [12]. It is therefore possible that individuals who show extreme effects of $\mathrm{ABx}$ treatment received additional doses outside of the time scale of this study. Without baseline microbiome 
diversity measures we are unable to capture such information. Similarly, estimating the effects of $\mathrm{ABx}$ on communities from data on microbes in isolation is, at best, approximate. A better understanding of how $\mathrm{ABx}$ affect complex communities is needed. Other limitations include our small sample size relative to the heterogeneity in the data. Future directions should capture variables such as the presence of brain metastases, tumor biomarkers such as tumor mutational burden and PD-1/ PDL-1 status, and outcome variables like ICI response or the number of tumor-infiltrating lymphocytes.

\section{Conclusions}

$\mathrm{ABx}$ and $\mathrm{CS}$, but not other medications known to affect the microbiome, are associated with reduced OS when administered near the start of ICI treatment. Our results show this finding several cancer types, and for several subclasses of these drugs. These results are consistent with a role of the microbiome in response to ICIs and identify clinical settings where the microbiome is likely to play the largest role, namely NSCLC, melanoma, RCC, HNSC, and bladder cancer. A clear understanding of which microbes are important for ICI responses and in what cancers will require the collection of microbiome samples across a wide variety of clinical settings. However, some information can be gathered by indirect means, which identifies the settings where the microbiome is likely to have the greatest effects. Medications that affect the microbiome given concomitantly with ICIs provide evidence for where microbes play a role. Further work is needed to identify which microbes are important and identify solutions to mitigate these effects and perhaps promote greater response to ICIs.

\section{Supplementary information}

Supplementary information accompanies this paper at https://doi.org/10. $1186 /$ s12885-020-06882-6.

Additional file 1: Figure S1. Causal diagram with references. Figure

S2. Number of medications prescribed across all cancers and the frequency of multiple medications. Figure S3. Number of antibiotics, separated by class, prescribed across all cancers and the frequency of multiple antibiotics. Figure S4. Number of corticosteroids, separated by class, prescribed across all cancers and the frequency of multiple antibiotics.

Additional file 2: Table S1. References for the antibiotic susceptibilities of bacterial taxa found to be enriched in responders or non-responders to $\mathrm{ICl}$ therapy

\section{Abbreviations}

ICls: Immune Checkpoint Inhibitors; OS: Overall Survival; ABx: Antibiotics; CS: Corticosteroids; CCl: Charlson Comorbidity Index; PS: Eastern Cooperative Oncology Group Performance Status; BMI: Body Mass Index; NSCLC: NonSmall Cell Lung Cancer; RCC: Renal Cell Carcinoma; HNSC: Head and Neck Squamous Cell Carcinoma

\section{Acknowledgements}

This work was performed on the Ohio Supercomputer Center servers (Owens, Pitzer; Project PA1490 awarded to Daniel Spakowicz). The authors would like to thank Stephanie Fortier (Division of Medical Oncology, The Ohio State University; ORCID 0000-0002-2380-2378) for critically reviewing and editing the manuscript.

\section{Ethic approval and consent to participate}

Data from this work were collected under an IRB-approved retrospective study (OSU-2016C0070, OSU-2017C0063).

\section{Authors' contributions}

$\mathrm{MH}, \mathrm{GT}, \mathrm{SP}, \mathrm{JTB}, \mathrm{CFV}, \mathrm{KLK}, \mathrm{SH}, \mathrm{JP}, \mathrm{DQ}, \mathrm{GAO}, \mathrm{MG}, \mathrm{CP}$ and DHO collected the data. $\mathrm{RH}, \mathrm{MM}, \mathrm{JB}, \mathrm{LW}, \mathrm{LL}, \mathrm{DPC}, \mathrm{DHO}$ and $\mathrm{DS}$ analyzed the data and generated the figures and Tables. DS, $\mathrm{RH}$ and $\mathrm{DHO}$ wrote the manuscript. All authors read and approved the final manuscript.

\section{Funding}

This project was supported by The Ohio State University Center for Clinical and Translational Science grant support (National Center for Advancing Translational Sciences, Grant 8UL1TR000090-05) and a P30 CCSG award (2 P30 CA016058-42). Daniel Spakowicz is supported by a Pelotonia New Investigator Award and Carolyn Presley is a Paul Calabresi Scholar supported by the OSU K12 Training Grant for Clinical Faculty Investigators (5 K12 CA133250-09). No funding body was involved in the design of the study or the collection, analysis, or interpretation of data, or in writing the manuscript.

Availability of data and materials

Scripts to reproduce all figures and analyses can be found at https://github. com/spakowiczlab/co-med-io.

Consent for publication

Not applicable.

\section{Competing interests}

$\mathrm{DHO}$ has a consulting or advisory role to L\&M Policy Research and AstraZeneca. DHO has research funding from Bristol-Myers Squibb, Palobiofarma, Merch Sharp \& Dohme and Genentech/Roche. DHO received travel, accommodations and/or expenses from AstraZeneca. DPC is on the advisory board of Abbvie, AMGEN, Ariad, AZ, Biocept, BI, BMS, Celgene, EMD Serono, Genentech, Gritstone, Helsinn, Inivata, Inovio, Janssen, Loxo Oncology, Merck, MSD, Pfizer, Roche, Takeda, and Trinity. DPC received honoraria from AstraZeneca and Nexus Oncology. DPC is a consultant for Abbvie, Agenus, Ariad, Celgene, Genentech, Incyte, Inivata, Janssen, Kyowa Kirin, Loxo, MSD, Novartis, Pfizer and Takeda.

\section{Author details}

${ }^{1}$ Division of Medical Oncology, Department of Internal Medicine, The Ohio State University Comprehensive Cancer Center, Columbus, OH, USA. ${ }^{2}$ Department of Biomedical Informatics, The Ohio State University College of Medicine, Columbus, OH, USA. ${ }^{3}$ Division of Hospital Medicine, Department of Internal Medicine, The Ohio State University Comprehensive Cancer Center, Columbus, USA

Received: 16 September 2019 Accepted: 21 April 2020

Published online: 06 May 2020

References

1. Gopalakrishnan V, Spencer CN, Nezi L, Reuben A, Andrews MC, Karpinets TV, et al. Gut microbiome modulates response to anti-PD-1 immunotherapy in melanoma patients. Science. 2018 Jan 5;359(6371):97-103.

2. Routy B, Le Chatelier E, Derosa L, Duong CP, Alou MT, Daillère R, et al. Gut microbiome influences efficacy of PD-1-based immunotherapy against epithelial tumors. Science. 2018;359(6371):91-7.

3. Matson V, Fessler J, Bao R, Chongsuwat T, Zha Y, Alegre M-L, et al. The commensal microbiome is associated with anti-PD-1 efficacy in metastatic melanoma patients. Science. 2018 Jan 5;359(6371):104-8.

4. Smith PM, Howitt MR, Panikov N, Michaud M, Gallini CA, Bohlooly-Y M, et al. The microbial metabolites, short-chain fatty acids, Regulate Colonic Treg Cell Homeostasis. Science. 2013 Aug 2;341(6145):569-73. 
5. Chen P-L, Roh W, Reuben A, Cooper ZA, Spencer CN, Prieto PA, et al. Analysis of immune signatures in longitudinal tumor samples yields insight into biomarkers of response and mechanisms of resistance to immune checkpoint blockade. Cancer Discov. 2016;6(8):827-37.

6. Limagne E, Richard C, Thibaudin M, Fumet J-D, Truntzer C, Lagrange A, et al. Tim-3/galectin-9 pathway and mMDSC control primary and secondary resistances to PD-1 blockade in lung cancer patients. Oncoimmunology. 2019;8(4):e1564505.

7. Huang AC, Orlowski RJ, Xu X, Mick R, George SM, Yan PK, et al. A single dose of neoadjuvant PD-1 blockade predicts clinical outcomes in resectable melanoma. Nat Med. 2019;25(3):454-61.

8. Kim Y-G, Udayanga KGS, Totsuka N, Weinberg JB, Núñez G, Shibuya A. Gut Dysbiosis promotes M2 macrophage polarization and allergic airway inflammation via fungi-induced PGE2. Cell Host Microbe. 2014 Jan 15;15(1):95-102.

9. Arbour KC, Mezquita L, Long N, Rizvi H, Auclin E, Ni A, et al. Impact of baseline steroids on efficacy of programmed cell Death-1 and programmed death-ligand 1 blockade in patients with non-small-cell lung Cancer. JCO. 2018 Oct;36(28):2872-8.

10. Rogers MAM, Aronoff DM. The influence of non-steroidal anti-inflammatory drugs on the gut microbiome. Clinical Microbiology and Infection. 2016 Feb 1:22(2):178.e1-178.e9.

11. Imhann F, Bonder MJ, Vich Vila A, Fu J, Mujagic Z, Vork L, et al. Proton pump inhibitors affect the gut microbiome. Gut. 2016 May;65(5):740-8.

12. Dethlefsen $L$, Relman DA. Incomplete recovery and individualized responses of the human distal gut microbiota to repeated antibiotic perturbation. Proc Nat Acad Sci. 2011;108(Supplement_1):4554-61.

13. Zhao S, Gao G, Li W, Li X, Zhao C, Jiang T, et al. Antibiotics are associated with attenuated efficacy of anti-PD-1/PD-L1 therapies in Chinese patients with advanced non-small cell lung cancer. Lung Cancer. 2019 Apr;130:10-7.

14. Elkrief A, El Raichani L, Richard C, Messaoudene M, Belkaid W, Malo J, et al. Antibiotics are associated with decreased progression-free survival of advanced melanoma patients treated with immune checkpoint inhibitors. Oncoimmunology. 2019;8(4):e1568812.

15. Chheda ZS, Sharma RK, Jala VR, Luster AD, Haribabu B. Chemoattractant receptors $B L T 1$ and $C X C R 3$ regulate antitumor immunity by facilitating CD8 ${ }^{+}$T cell migration into tumors. J. 2016;197(5):2016-26.

16. Wu T, Yang L, Jiang J, Ni Y, Zhu J, Zheng $X$, et al. Chronic glucocorticoid treatment induced circadian clock disorder leads to lipid metabolism and gut microbiota alterations in rats. Life Sci. 2018 Jan;192:173-82.

17. Garant A, Guilbault C, Ekmekjian T, Greenwald Z, Murgoi P, Vuong T. Concomitant use of corticosteroids and immune checkpoint inhibitors in patients with hematologic or solid neoplasms: a systematic review. Crit Rev Oncol Hematol. 2017 Dec;120:86-92.

18. Lozupone CA, Stombaugh Jl, Gordon Jl, Jansson JK, Knight R. Diversity, stability and resilience of the human gut microbiota. Nature. 2012 Sep 13; 489(7415):220-30.

19. González-Amaro R, Sánchez-Madrid F. Drugs, inflammation and cell adhesion receptors. Expert Opin Pharmacother. 2001;2(1):3-17.

20. Maier L, Pruteanu M, Kuhn M, Zeller G, Telzerow A, Anderson EE, et al. Extensive impact of non-antibiotic drugs on human gut bacteria. Nature. 2018 Mar;555(7698):623-8.

21. Fucà G, Galli G, Poggi M, Lo Russo G, Proto C, Imbimbo M, et al. Modulation of peripheral blood immune cells by early use of steroids and its association with clinical outcomes in patients with metastatic non-small cell lung cancer treated with immune checkpoint inhibitors. ESMO Open. 2019 Feb; 4(1):e000457.

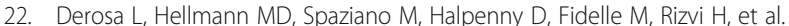
Negative association of antibiotics on clinical activity of immune checkpoint inhibitors in patients with advanced renal cell and non-small-cell lung cancer. Ann Oncol. 2018 Jun 1;29(6):1437-44.

23. Hussain M, Javeed A, Ashraf M, Al-Zaubai N, Stewart A, Mukhtar MM. Nonsteroidal anti-inflammatory drugs, tumour immunity and immunotherapy. Pharmacol Res. 2012 Jul;66(1):7-18.

24. Wang D, DuBois RN. Prostaglandins and cancer. Gut. 2006 Jan;55(1):115-22.

25. Jackson MA, Goodrich JK, Maxan M-E, Freedberg DE, Abrams JA, Poole AC, et al. Proton pump inhibitors alter the composition of the gut microbiota. Gut. 2016 May;65(5):749-56.

26. Boursi B, Mamtani R, Haynes K, Yang Y-X. Recurrent antibiotic exposure may promote cancer formation - another step in understanding the role of the human microbiota? Eur J Cancer. 2015 Nov;51(17):2655-64.
27. Lidar M, Giat E, Garelick D, Horowitz Y, Amital H, Steinberg-Silman Y, et al. Rheumatic manifestations among cancer patients treated with immune checkpoint inhibitors. Autoimmun Rev. 2018 Mar;17(3):284-9.

28. Leonard J, Marshall JK, Moayyedi P. Systematic review of the risk of enteric infection in patients taking acid suppression. Am J Gastroenterology. 2007 Sep;102(9):2047-56.

29. Park JH, MCMillan DC, Horgan PG, Roxburgh CS. The impact of antiinflammatory agents on the outcome of patients with colorectal cancer. Cancer Treat Rev. 2014 Feb;40(1):68-77.

30. Huang EY, Inoue T, Leone VA, Dalal S, Touw K, Wang Y, et al. Using corticosteroids to reshape the gut microbiome: implications for inflammatory bowel diseases. Inflamm Bowel Dis. 2015 May;21(5): 963-72.

31. Jala VR, Bodduluri SR, Satpathy SR, Chheda Z, Sharma RK, Haribabu B. The yin and yang of leukotriene B4 mediated inflammation in cancer. Semin Immunol. 2017;33:58-64.

32. Zitvogel L, Ayyoub M, Routy B, Kroemer G. Microbiome and Anticancer Immunosurveillance. Cell. 2016 Apr 7;165(2):276-87.

33. Csardi G, Nepusz T. The igraph software package for complex network research. InterJournal. 2006;Complex Systems:1695.

34. Textor J, Zander B van der. dagitty: Graphical Analysis of Structural Causal Models [Internet]. 2016. Available from: https://CRAN.R-project. org/package $=$ dagitty

35. Harris PA, Taylor R, Thielke R, Payne J, Gonzalez N, Conde JG. Research electronic data capture (REDCap)--a metadata-driven methodology and workflow process for providing translational research informatics support. J Biomed Inform. 2009 Apr;42(2):377-81.

36. Charlson M, Szatrowski TP, Peterson J, Gold J. Validation of a combined comorbidity index. J Clin Epidemiol. 1994;47(11):1245-51.

37. Kassambara A, Kosinski M. survminer: Drawing Survival Curves using "ggplot2" [Internet]. 2018. Available from: https://CRAN.R-project.org/ package $=$ survminer

38. Core R. Team. R: a language and environment for statistical computing [internet]. Vienna, Austria: R Foundation for statistical. Computing. 2017; Available from: https://www.R-project.org/.

39. Zeileis A, Hothorn T. Diagnostic checking in regression relationships. 2002 [cited 2017 May 8]; Available from: http://idg.pl/mirrors/CRAN/web/ packages/Imtest/vignettes/Imtest-intro.pdf.

40. Therneau TM. A package for survival analysis in S [internet]. 2015. Available from: https://CRAN.R-project.org/package=survival.

41. Therneau TM, Grambsch PM. Modeling survival data: extending the cox model. New York: Springer; 2000.

42. Wickham H. ggplot2: elegant graphics for data analysis [Internet]. Springer Science \& Business Media; 2009 [cited 2015 Dec 14]. Available from: https://books.google.com/books?hl=en\&lr=\&id=bes-AAAAQBAJ\&oi= fnd\&pg =PR5\&dq=ggplot2\&ots=SAc_MyOSQR\&sig=kx|4eEM08tk2hMmUo5 UgBJQE4AQ.

43. Wishart DS, Feunang YD, Guo AC, Lo EJ, Marcu A, Grant JR, et al. DrugBank 5.0: a major update to the DrugBank database for 2018. Nucleic Acids Res. 2018;46(D1):D1074-82

44. Gehlenborg N. UpSetR: a more scalable alternative to Venn and Euler diagrams for visualizing intersecting sets [internet]. 2019. Available from: https://CRAN.R-project.org/package=UpSetR.

45. Friedman J, Hastie T, Tibshirani R. Regularization paths for generalized linear models via coordinate descent. J Stat Softw. 2010:33(1):1-22.

46. Simon N, Friedman J, Hastie T, Tibshirani R. Regularization paths for Cox's proportional hazards model via coordinate descent. J Stat Softw. 2011;39(5):1-13.

47. Charlson ME, Charlson RE, Peterson JC, Marinopoulos SS, Briggs WM, Hollenberg JP. The Charlson comorbidity index is adapted to predict costs of chronic disease in primary care patients. J Clin Epidemiol. 2008 Dec; 61(12):1234-40

48. Ricciuti B, Dahlberg SE, Adeni A, Sholl LM, Nishino M, Awad MM. Immune Checkpoint Inhibitor Outcomes for Patients With Non-Small-Cell Lung Cancer Receiving Baseline Corticosteroids for Palliative Versus Nonpalliative Indications. JCO. 2019;19:00189.

49. Pinato DJ, Howlett S, Ottaviani D, Urus H, Patel A, Mineo T, et al. Association of Prior Antibiotic Treatment With Survival and Response to Immune Checkpoint Inhibitor Therapy in Patients With Cancer. JAMA Oncol. 2019. 
50. Wilson BE, Routy B, Nagrial A, Chin VT. The effect of antibiotics on clinical outcomes in immune-checkpoint blockade: a systematic review and metaanalysis of observational studies. Cancer Immunol Immunother. 2020 Mar 1; 69(3):343-54.

51. Pérez-Cobas AE, Gosalbes MJ, Friedrichs A, Knecht H, Artacho A, Eismann K, et al. Gut microbiota disturbance during antibiotic therapy: a multi-omic approach. Gut. 2013 Nov 1;62(11):1591-601.

52. Nandi T, Lee IR, Ghosh T, Ng AHQ, Chng KR, Li C, et al. Gut microbiome recovery after antibiotic usage is mediated by specific bacterial species. bioRxiv. 2018;19:350470.

\section{Publisher's Note}

Springer Nature remains neutral with regard to jurisdictional claims in published maps and institutional affiliations.

Ready to submit your research? Choose BMC and benefit from:

- fast, convenient online submission

- thorough peer review by experienced researchers in your field

- rapid publication on acceptance

- support for research data, including large and complex data types

- gold Open Access which fosters wider collaboration and increased citations

- maximum visibility for your research: over $100 \mathrm{M}$ website views per year

At $\mathrm{BMC}$, research is always in progress.

Learn more biomedcentral.com/submissions 